\title{
VARIAÇÃO TEMPORAL DA ABUNDÂNCIA E COMPOSIÇÃO ESPECÍFICA DA MACROFLORA ASSOCIADA A UMA POPULAÇÃO DE SARGASSUM (FUCOPHYCEAE) DO LITORAL SUL DE PERNAMBUCO, BRASIL.
}

\author{
ANDRÉS O. MANSILLA M. \& SONIA M. B. PEREIRA
}

1) pantamento de Biologia, Universidade Federal Rumal cle Pernambuco, Av. Dom Mamuel de Medeiros s/n, Dois Irmãos, 52171-900, Recife, PE, Brasil

\begin{abstract}
Scasonal variations in abundance and composition of the macrof lora associated to Sargassum (Fucophyceae) of the South littoral of Pernambuco, Brazil). The border community of Sargassum from the rock shore of Pedra do Xaréu, locataed $30 \mathrm{~km}$ south of Recife city (PE-Brazil) was characterized in terms of abundance and relative frequency. Samples were collected with a plastic ring of $50 \mathrm{~cm}^{2}$ of area, throwed 30 times in aleatory way in January, February (dry season), and June, July (rainy scason). The dominant specie was Sargassum polyceratium Montagne, that presented an average biomass of $445.15 \pm 138.47$ (dry scatson) and $38.3 \pm 104.65$ (rainy season) (dry wcight, $\mathrm{g} / \mathrm{m} 2$ ). The best represented Division was Rhodophyta (46.15\%), followed by Fucophyceac $(38.46 \%)$ and Chlorophyta (15.39\%). S. polyceratium was preferable host to the epiphytic macroalgae, which show low ability to colonize hard substratum, contrasting with the competitive ability of Sargassum. Hydrological parameters didn't present expressive variations, although wave exposure seems to be the main parameter to control the studied population.

Resumo - (Variação temporal da abunclância e cla composição específica da macrof lora associada a uma população de Sargassum (Fucophyceae) do litoral sul de l'ermanbuco, Brasil). A comunidade da margem de Sargassum do costão rochoso de Pedra dı Xaréu, localizado a $30 \mathrm{~km}$ de Recife, foi caracterizada em termos de abundância e frequência relativa. Para amostragem, utilizou-se una circunferência de plástico de $50 \mathrm{~cm}^{2}$ de área, lançada 30 vezes de forma aleatória na área, os lançamentos foram posicionados de acordo com coordenadlas aleatórias, nos meses de janeiro, fevereiro (época seca) e junho, julho (época chuvosa). A espécie clominante foi Sargassum polyceratium_Montagne, que apresentou biomassa médias de 445, 15 $\pm 138,47$ (período seco) c $38.3 \pm 104,65$ (período chuvoso). As macroalgas mellır representadas foram as da Divisão Rhodophyta $(46,15 \%)$, seguiclas pelas Fucophyceae $(38,46 \%)$ e Chlorophyta (15,39\%). S. polyceratium apresentou-se como um bom hospecleiro de macroalgas epífitas, as quais parecem apresentar pouca habilidade para colonizar substrato rochoso, em contraste com as características de dominante competitivo e colonizador oportunista de Sargassum. Os parâmetros hidrológicos não apresentalam variações expressivas, mas a exposição as ondass parece ser o parâmetro que regula a dinâmica da população estudada.
\end{abstract}

Keywords: Rock shore, Ecology, Pernambuco, Brazil

\section{Introdução}

No Brasil, os costões rochosos são característicos do litoral sudeste c sul clo país, enquanto que no Nordeste, os recifes de arcnito são as formações mais representativas. Porém no litoral Sul do Estado de Pernambuco, ocorrem trechos de costões rochosos e matacões de origem vulcânica semelhante àqueles das regiões sul e sudeste do país, como por exemplo os costões de pedra do Xaréu, Cabo de Santo Agostinho e os da Ilha de Santo Alcixo.

Numcrosos trabalhos, descrevendo a distribuição de organismos na região entremarés em costões rochosos, foram realizados principalmente para o litoral clo Estado São Paulo, destacando-se os de Nonato e Pèrés (1961), Oliveira Filho e Mayal (1976) e Paula \& Oliveia (198()); para o Arquipélago de Fernando de Noronha, cita-se o de Eston et al. (1986). Para o litoral sul, de Pernambuco o único trabalho realizado foi o de Accioly (1992). Em todos estes estudos foi observada a presença de um local caracterizado pela ocorrência de espécies do gênero Sargassum, localizada na zona infralitorânea. Segundo Oliveira Filho e Mayal (1976), podem ocorrer nestas faixas plantas de Sargassum com características morfológicas distintas, espécies diferentes de Sargassum_ou inclusive esta zona pode estar substituída por algas de outros gêneros, dependendo principalmente da ação das ondas existente na área ou das latitudes nas quais ocorrem estas formações (Lewis 1964, Oliver et al. 1966, Nibakken 1969, Shepinerd \& Womersley 1970, Russell 1977, Chapman 1979, Buschmann 1992).

Accioly (1992), trabalhando sobre metodologia de amostragem e zonação das comunidades bentônicas no costão rochoso de Pedra do Xaréu, verifïcou a ocorrência de 64 unidades taxonômicas operacionais, reco- 
nhecendo, apenas, três comunidades bem definidas: margem crostosa dominada por cianofíceas, pardas crostosas, Chtamalus bisinuatus_Pilsbry, 1916 e Littorina ziczac_Gmelin, 1791, com baixos índices de riqueza, diversidade e equitabilidade; tapete de algas, dominada por algas bem distribuídas e pouco desenvolvidas, com indices de diversidade bastante elevados e a margem de Sargassum que apresentou-se formada por uma densa população de $S$. polyceratium, com outras unidades taxonômicas esparsas, principalmente Anadyomene stellata (Wulfen) C. Agardh e_Laurencia papillosa (C. Agardh) Creville.

Tenclo em vista a importância ecológica do gênero Sargassum nos costões rochosos, tem este trabalho o objetivo de fornecer dados sobre a variação temporal cla biomassa e composição específica da macroflora associada à comunidade da margem de Sargassum.

\section{Material e Métodos}

A praia de Pedra do Xaréu situa-se no Município do Cabo, distante $30 \mathrm{~km}$ ao Sul da cidade do Recife $\left(8^{\circ} 18^{\prime} 14^{\prime \prime} \mathrm{S}\right.$ e $\left.34^{\circ} 56^{\prime} 45^{\prime \prime} \mathrm{W}\right)$, apresenta aproximadamente $1 \mathrm{~km}$ de extensão, onde ocorrem formações rochosas sol) a ação de grande exposição de ondas, (Figura 1). Estes costões são de origem vulcânica formados há aproximadamente 120 a 70 milhões de anos, sendo sua composição de traquito com abundante silica e cscasso quartzo Newmann (com. pessoal) .

No costão de Pedra do Xaréu, foi escolhida uma comunidade dominada por $S$. polyceratium, na qual foi delimitada uma faixa de $15 \mathrm{~m} \times 4 \mathrm{~m}$, para a realização do trabalho. Para amostragem, utilizou-se uma circunferência de plástico de $50 \mathrm{~cm}^{2}$ de área, lançada 30 vezes de forma aleatória, os lançamentos foram posicionados de acordo com coordenadas aleatórias; o processo foi repetido, nos meses de janeiro, fevereiro (época seca) c junho, julho (época chuvosa), sempre nas baixamares, no período de lua nova. A eficiência da unidade amostral, assim como o número de amostras necessárias para representar a comunidade, foram testadas exaustivamente em coletas pré-experimentais, através de curvas espécies/áreas, nas quais foram determinados os pontos Molinier 20/1 e 20/2 (Boudouresque, 1971) e realizados os cálculos de eficiência relativa (Accioly, 1992). Com base nesta metodologia, foi observada a lreqüência de ocorrência (\%) das espécies nas amostras e determinada a biomassa (peso seco) de acordo com De Wreede (1985). A diversidade específica foi calculada pelo índice de Shannon (1948).

lioram realizadas também medições de parâmetros hidrológicos como: 1) oxigênio dissolvido, aplicandose o tradicional método de Winkler descrito por Strickland \& Parson (1965); 2) potencial hidrogeniônico, utilizando-se um pH-metro eletrônico, tipo Metronal120, seguindo-se a metodologia descrita em Unesco (1973); 3) salinidade, pelo método indireto de MorhKnudsen, descrito por Strickland \& Parson (1965); 4) temperatura, através de um termômetro de inversão protegido; 5) teores de nutrientes inorgânicos, utilizando a metodologia descrita por Strickland \& Parson (1965) e Unesco (1973). O material foi coletado com garrafas ocenográficas de Nansen, na camada superficial a dois metros do costão estudado.

\section{Resultados e Discussão}

As macroalgas melhor representadas foram as da Divisão Rhodophyta $(46,15 \%)$, seguidas pelas Phaeophyta $(38,46 \%)$ e Chlorophyta (15,39\%) (Tabela 1). A clorofícea Cladophoropsis membranacea (C. Agardh) Börgesen ocorreu como epilítica, enquanto que Anadyomene stellata (Wulfen) C. Agardh sempre ocorreu como epífita do apressório de $S$. polyceratium. Algumas rodofíceas, como Dipterosiphonia dendritica_(C Agardh) Schmitz in Engler et Prantl, Hypnea musciformis_(Wulfen in Jaquin) Lamouroux e Fosliella sp. apresentaram-se sempre, na condição de epífitas de $S$. polyceratium_ou de outras macroalgas, enquanto que Amansia multifida Lamouroux, Laurencia papillosa (C. Agardh) Greville e Laurencia sp. ocorreram como epilíticas. Situação semelhante foi observada para as espécies de fucofíceas, na qual Dictyopteris delicatula Lamouroux e Dictyota dichotoma (Hudson) Lamouroux apresentaram-se sempre como epífitas, excetuando-se Padina gymnospora (Kützing) Sonder, Spatoglossum schroederi_(C. Agardh) Kützing e $S$. polyceratium que ocorreram como epilíticas. As cianofíceas e rodofíceas crostosas estiveram sempre presentes recobrindo o substrato. Eston \& Bussab (1990), num estudo sobre a dominância ecológica de Sargassum stenophyllum (Mertens) Martius, em costões rochosos do sudeste brasileiro, observaram a ocorrência de Dictyopteris delicatula e D. plagiograma, as quais se caracterizaram por apresentar pouca habilidade para se fixarem em substrato rochoso, permitindo portanto, o estabelecimento de Sargassum. Largo et al. (1994), estudaram as mudanças sazonais em comunidades dominadas por Sargassum polycystum C. Ag. e Sargassum siliquosum J. Ag., mas não fizeram referência à flora associada de macroalgas.

A freqüência das macroalgas (Tabela 1), na comunidade dominada por Sargassum polyceratium, de uma maneira geral apresentou pouca variação mensal, tanto no período seco como no chuvoso, sendo que Sargassum polyceratium, Dipterosiphonia dendritica_e Fosliella sp. foram as espécies mais frequentes.

Os grandes e fortes apressórios de $S$. polyceratium, parecem impedir a ocorrência de uma maior quantida- 


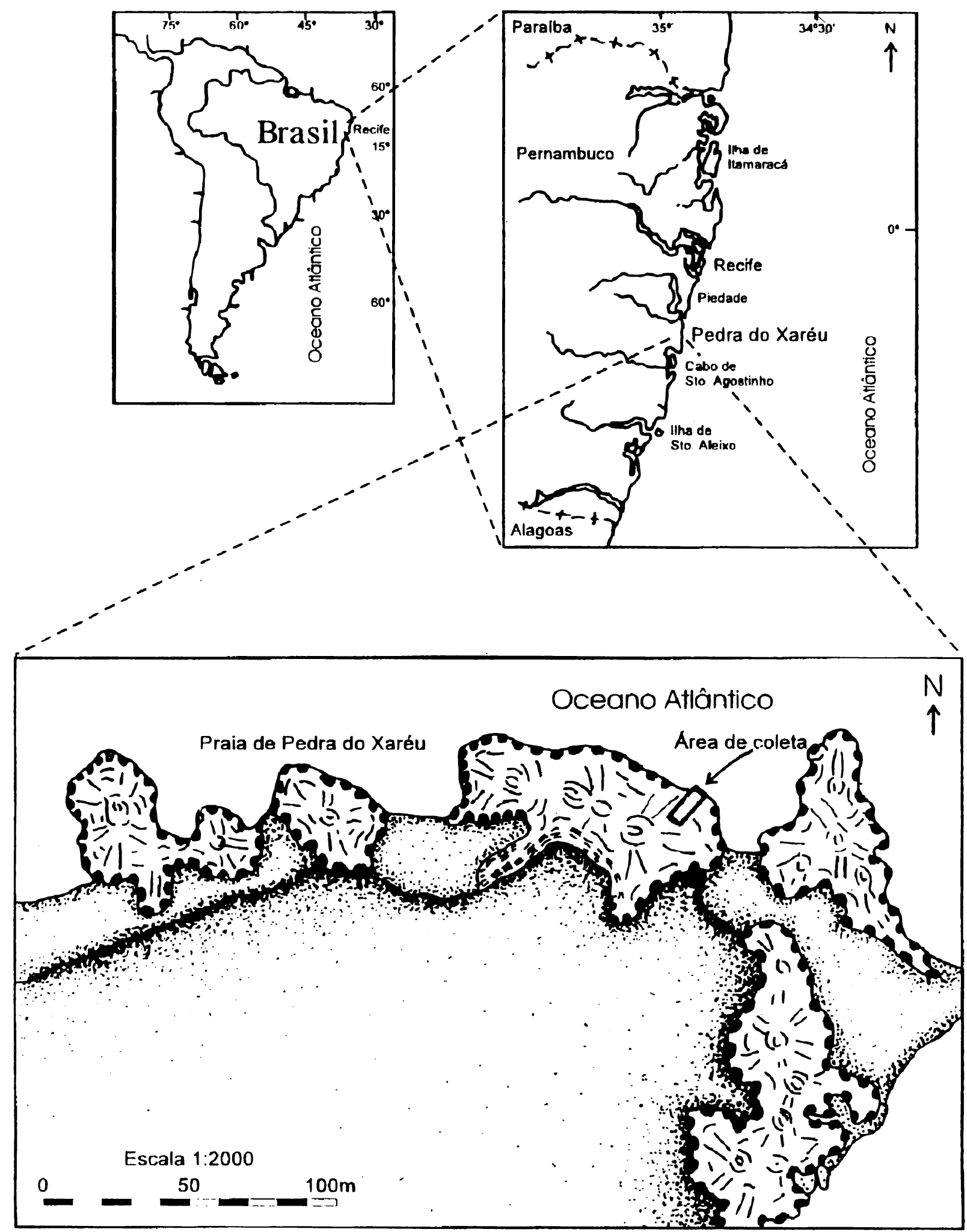


Tahela 1. Dados de frequência relativa (\%), das macroalgats coletadas na comunidade de Sargassum, nos meses de janeiro e fevereiro/92 (períselo seco) e junho e julho/92 (período chuvoso), no costão rochoso de l'eclra do Xaréu - Cabo - PE, Brasil.

\begin{tabular}{|c|c|c|c|c|c|c|}
\hline & JANEIRO & FEVEREIRO & VALOR MÉDIO & JUNHO & JULHO & VALOR MÉDIO \\
\hline \multicolumn{7}{|l|}{ CIIIOOROIHYTA } \\
\hline 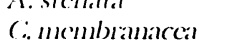 & - & - & - & 0,1 & 0,13 & 0,11 \\
\hline \multicolumn{7}{|l|}{ PIIAEOPHYTA } \\
\hline I). (kelicatulat & 1,8 & 0,5 & $1,15 \pm 0,91$ & 0,1 & 0,3 & $(0,2 \pm(), 14$ \\
\hline 1). dicheromal & - & ()$, 12$ & 0,06 & - & - & - \\
\hline 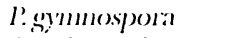 & 4 & - & 2,0 & - & 0,64 & 0,32 \\
\hline S. polyceratim & 341 & 547 & $445,15 \pm 1.38,47$ & 309 & 457 & $38.3 \pm 104,65$ \\
\hline Sischuecleri & - & - & - & - & 0,07 & $0,(0) 35$ \\
\hline \multicolumn{7}{|l|}{ RHODOPHYTA } \\
\hline 1. multifïlla & - & 0,05 & 0,025 & 0,1 & 0,08 & $0,09 \pm 0,014$ \\
\hline 1). denclitical & - & - & - & - & - & - \\
\hline Loslicllatsp. & - & - & - & - & - & -- \\
\hline II. musciformis & $(0,2$ & 0,58 & $(0,39 \pm(), \underline{2} 6$ & 0,2 & - & ()$, 1$ \\
\hline L. papillosa & 12 & 0,1 & $(0,05 \pm 8,41$ & & 0,03 & 0,015 \\
\hline Lallu(enciasp). & 42 & $(0,1$ & $21,05 \pm 29,62$ & 0,3 & 0,62 & $0,46 \pm 0,22$ \\
\hline TOTAL & 101 & 548,56 & $474,78 \pm 104,34$ & 310 & 459,52 & $384,76 \pm 105,72$ \\
\hline
\end{tabular}

de e diversidade de algas cpilíticas. Estes comentários coincidem com os de Eston \& Bussab (199)), ao se referirem que espécies de Sargassum podem ser consideradas como dominantes competitivos e colonizadores oportunistas.

A biomassa média total para o período seco foi de (948,93 $\mathrm{g} \mathrm{m}^{\circ 2}$, decrescendo para 769,52 $\mathrm{g} \mathrm{m²}$ no período chuvoso. Observou-se que $S$. polyceratium, contribuiu de uma maneira preponderante na biomassa total da comuniclade estudada. Outras espécies, embora pouco firepuentes como_Laurencia papillosa e Laurencia sp. também apresentaram biomassas considerávcis (Tabcla 2).

Talucla 2. Dados de biomasssa (g peso seco $\mathrm{m}^{20}$ ), clas macroalgas coletaclas na comunidade de Sargassum, nos meses de janciro e feverciro/!)2 (período seco) e junho e julho/92 (período chuvoso), un costaio rochoso de P'edra clo Xaréu - Cabo - PE, Brasil.

\begin{tabular}{|c|c|c|c|c|}
\hline & JANEIRO & FEVEREIRO & JUNHO & JULHO \\
\hline \multicolumn{5}{|l|}{ (:HLOROPIYTA } \\
\hline A. stcllatla & 2.29 & 2.15 & 3.01 & 5.8 \\
\hline Comcmlnanaceal & - & - & 0.13 & 1.93 \\
\hline \multicolumn{5}{|l|}{ PHAEOPHYTA } \\
\hline 1). clelicatulial & $3 .(05)$ & 1.07 & 4.81 & $7 .(09)$ \\
\hline D. dichotoma & - & 2.15 & - & -- \\
\hline l. gymmospotat & 1.52 & - & - & 0.64 \\
\hline S.polvceratimn & 21.13 & 31.1 & 18.1 & 18.1 \\
\hline S. schiosederi & - & - & - & 1.29 \\
\hline \multicolumn{5}{|l|}{ RIIODOPHYTA } \\
\hline A. multifïlar & - & 1.07 & 1.8 & 1.39 \\
\hline D. dendritica & 9.9 & 1.07 & 16.7 & 18.1 \\
\hline Fosliclla spo. & 20.6 & $1(i .1$ & 17.5 & 18.1 \\
\hline H. musciformis & 6.1 & 8.6 & 3.61 &.- \\
\hline L. pippillosal & 1.07 & 1.07 & - & 0.64 \\
\hline Lauluencia sp). & 5.34 & 3.22 & 4.21 & 5.16 \\
\hline
\end{tabular}

O valor médio da biomassa total de $S$. polyccratium $\left(413,5 \mathrm{~g} \mathrm{~m}^{2} \pm 109,3\right)$, manteve-se entre os valores médios obticlos por outros autores, como Paula (1978), que cucontrou médias de 389, $2 \mathrm{gm}^{-2}$ para espécies deste yênero, no costão batido do Município de Ubatuba -
São Paulo, por Eston et al. (1986) de 230 a $480 \mathrm{gm}^{-2}$, para costões do Arquipélago de Fernando de Noronha, embora $\mathrm{cm}$ todos estes trabalhos tenham sido utilizadas unidades amostrais diferentes. Carvalho (1983), trabalhando num complexo recifal do litoral paraibano, obteve biomassas que variaram entre 532,38 a $687,56 \mathrm{gm}^{-2}$.

Acredita-se que os elevados valores obtidos por Carvalho (1983), sejam justificáveis porque, em formações recifais os espécimens de Sargassum, são encontrados em locais protegidos, representados pelas poças recifais, nas quais, devido à moderada exposição as ondas existente, ocorrem exemplares mais desenvolvidos. Paula \& Oliveira Filho (1982), observaram os mesmos padrões numa população de Sargassum cymosum C. Agardh, nos costões rochosos de Ubatuba, São Paulo, nos quais a exposição à ação das ondas resultou numa diminuição no tamanho das plantas.

Tabela 3. Dados hichrológicos medidos no costão rochoso de Pedra do Xaréu-PE, Brasil.

\begin{tabular}{|c|c|c|c|c|}
\hline & Janeiro/92 & Fevereiro/92 & Junho/92 & Julho/92 \\
\hline Temperatura ( ${ }^{\circ} \mathrm{C}$ ) & 27,00 & 29,30 & 28,00 & 28,00 \\
\hline $\mathrm{pH}$ & 8,49 & 8,27 & 8,10 & $8, \underline{20}$ \\
\hline Salinichale (\%o) & 36,34 & 34,62 & 34,00 & 36,00 \\
\hline Oxigênio $\left(m l^{-1}\right)$ & 6,02 & 5,91 & 4,15 & 5,31 \\
\hline Nitrito $\left(\mu\right.$ moll $\left.^{-1}\right)$ & - & 0,070 & - & 0,01 \\
\hline Nitrato $\left(\mu\right.$ moll $\left.^{-1}\right)$ & - & 2,39 & - & 2,90 \\
\hline Fosfato $\left(\mu\right.$ moll $\left.^{-1}\right)$ & - & 0,440 & - & 0,33 \\
\hline
\end{tabular}

A diversidade específica de Shannon, baseada nos dados de freqüência, apresentou valores de 3,111 bits. ind $^{-1}$ (período chuvoso) e 3,048 bits. ind ${ }^{-1}$ (período seco) (Tabela 4). Acredita-se que o pequeno número de espécies encontrado na comunidade da margem de Sargassum scja devido a uma baixa adaptabilidade que certas algas apresentariam para resistir a uma forte arreben- 
lação, relacionada talvez a um precário sistema de fixação, pouca flexibilidade ou mesmo falta de adaptações reprodutivas, como por exemplo, a baixa habilidade de algumas espécies do gênero Dictyopteris, para colonizar substrato rochoso (Eston \& Bussab, 1990).

Tialsela 4 - Diversidacle específica (bits.ind.-1), nos meses de janeiro e fevereiro/92 ( período seco) e junho e julho/92 (período chuvoso), no costĩo rochoso de Pedra do Xaréu- Cabo- PE, Brasil

\begin{tabular}{lll}
\hline & Diversidade específica & (bits.ind.-1) \\
\hline Janciro & & 3,003 \\
Fererciro & & 3,093 \\
Junhor & & 3,093 \\
Julhw & 3,133 \\
\hline
\end{tabular}

A ocorrência de cpífitas de $S$. polyceratium, cntre estas Dipterosiphonia dendritica, Fosliella sp., Hypnea musciforminis, Dictyopteris delicatula, Dictyota dichotoma_e Anadyomene stellata, demonstra a sua importância ecológica como hospedeiro, proporcionando microhabitats que permitem o descnvolvimento de outros organismos. Este fato tem sido mencionado por Chan (1981), Masunari (1989) entre outros.

O costão rochoso de Pedra do Xaréu, apresentou scmpre uma forte arrebentação. Esta afirmação se rellete na ocorrência de $S$. polyceratium_com característicals morlológicas típicas de espécies deste gênero, quando coletadas em costões fortemente expostos, como por exemplo, exemplares pequenos (em torno de $10 \mathrm{~cm}$ de altura), apressório bem desenvolvido e fortemente aderido, ausência de flutuadores e ocorrência de plantas hermafroditas durante todo o período de coleta, correspondendo aos comentários de Paula \& Oliveira Filho (1982).

A comunidade estudada caracterizou-se pela ocorrência de organismos $100 \%$ marinhos. A salinidade não variou de uma mancira expressiva, mas é importante destacar que o mês de fevereiro apresentou o menor registro, isto é, $34,36 \%$, devido a uma elevada e singular precipitação $(349 \mathrm{~mm})$. Deste modo é importante destacar, que no costão de Pedra do Xaréu, mesmo nos meses de maior pluviometria, a salinidade se manteve com índices elevados. O oxigênio dissolvido apresentou variações pouco pronunciadas durante o período de estudo, observando-se sempre um índice de saturaÇĩo superior a 100, demonstrando que a área não aprescnta índices críticos de poluição e que, devido à forte arebentação existente na área, a oxigenação é elevada e constante. Tanto o potencial hidrogenioninico, como os nutrientes apresentaram variações consideradas normais para cstas latitudes e que a exposição as ondas deve aletar a dinâmica desta comunidade. A temperatura não apresentou variações expressivas, portanto, parece não interferir na comunidade, como ocorre em regiões de maiores latitudes, nas quais existe uma correlação entre a temperatura e a distribuição de espécies (Guimaraens \& Coutinho, 1996).

\section{Agradecimentos}

Ao CNPq, pelo apoio a S. M. B. Pereira através de Bolsa de Pesquisa. Ao Dr. Edison José de Paula, pela colaboração na identificação de $S$. polyceratium e ao Dr. Eurico Cabral de Oliveira Filho, pela revisão do manuscrito e valiosas sugestões.

\section{Referências}

ACCIOLY, M DA C. de 1992. Metodologia de amostragem e zonação das connuniclades bentônicas do costão rochoso da praia de Peclra do Xaréu-Estado de Pernambuco (Brasil). Dissertação de Mestrado, Universidade Federal Rural de Pernambuco. Recife.

BOUDOURESQUE, C. F. 1971. Contribution à l'étude phytosociologique des peuplements algaux des côtes varoises. Vegetatio 22(1/ 3): 83-184.

BUSCHMANN, A. H. 1992. Algal communitics of a wave-protected intertidal rocky shore in Southern Chile. In U. Seeliger (ed.). Communities of Latin America coast plants. Academic Press. San Diego, p. 91-103.

CARVALHO, F. A. 1983. Biomassa bêntica do complexo recifal clo litoral clo Estado da Panaibal, com ênfase nas maciófiltas. Tese de Doutorado, Instituto Oceanográfico, Universidade de São Paulo. São Paulo.

CHAN, M. do S. 1981. Species diversity and zonation of epiphytes on two species of Sargassum. Proceeding of the Ath International Coral Reef Symposium. Manila. Vol. 2.

CHAPMAN, A. R. O. 1979. Biology of seaweeds: level of organization. E. Arnold. Halifax.

DE WREEDE, R. E. 1985. Destructive (harvest) sampling. In M. M. Littler \& D. S. Littler (eds). Handlbook of phycological methods Macroalgae. Cambridge University Press. Cambriclge, p. 147-175.

ESTON, V. R. \& BUSSAB, W. O. 1990. An experimental analysis of ecological clominance in a rocky subticlal macroalgal community.J. Exp. Mar: Biol. Ecol. 136: 179-195.

ESTON, V. R., MIGOTTO, A. E., OLIVEIRA FILHO, E. C., RODRIGUES, S. A. \& FREITAS, J. C. 1986. Vertical distribution of benthic marine organisms on rocky coasts of the Fernando de Noronha Archipelago (Brazil). Bol. Inst. Oceanogri: São Paulo 34: $37-53$.

GUIMARAENS, M. A. \& COUTINHO, R. 1996. Spatial and temporal variation of benthic marine algae at the Cabo Frio upwelling region, Rio de Janeiro, Brazil. Aquatic Bot. 52: 283-299.

LARGO, D. B., OHONO, M. \& CRITCHLEY A. T. 1994. Seasonal changes in the growth and reproduction of Sargassum polycystum C. Ag. and Sargassum siliquosum J. Ag. (Sargassaccae, Fucales) from Liloan, Cebu, in Central Plilippines. Jpn. J. Phycol. 42: 53-61.

LEWIS, J. R. 1964. The ecology of rocky shores. English University Press. London.

MASUNA RI, S. 1989. Ecologia da comunidades fitais. I Simpósio de Ecossistemas cla Costa Sul-Sudeste Brasileira, vol. 1, p. 195-253.

NIBAKKEN, J. W. 1969. P'recarthquake interticlal ccology of Three Saint Bay, Kodiak Island, Alaska. Biol. Pap. V. Alaska 9: 117p.

NONATO, E. \& PÉRÈS, J. M. 1961. Observation sur quelques peuplements intertidaux de substrat dur dans la régrion d'Ubatuba (État de São P’aulo). Cahier's Biol. Mas: 2: 263 - 270. 
(OLIVEIRA FILHO, E. C. DE. \& MAYAL, M. 1976. Seasonal distribution of intertidal organisms at Ubatuba, São Paulo (Brazil). Rev. Bras. Biol. 36: 305-316.

OLIVER, R. R., PATERNOSTER, E. K. DE, BASTIDA, R. 1966. Estudios biocenóticos en las costas de Chubut (Argentina), I. Zonación biocenológica de Puerto Pardelas (Golfo Nuevo). Bol. Inst. Biol. Mar: 10:1-74.

PAULA. E. J. \& OLIVEIRA, E.C. 1980. Aspectos fenológicos de duas populações de Sargassum (Phaeophyta - Fucales) do litoral de São Paulo. Bol. Bot. Univ. São Paulo 8: 21-39.

PAULA, E. J. \& OLIVEIRA FILHO, E. C. 1982. Wave exposure and ccotypical differentiation in Sargassum cymosum. Phycologia. 2) (2): $145-153$

RUSSELL, G. 1977. Vegetation on rocky shores at some North Irish
Sea sites. J. Ecol. 65:485-495.

SHANNON, C. E. 1948. A mathematical theory of communication. Bol. Syst. Tech. J. 27: 379-423.

SHEPINERD, S. A. \& WOMERSLEY, H. B. S.. 1970. The sublittoral ecology of West Island, South Australia, I Environmental features an algal ecology. Trans. Roy. Soc. S. Austr: 94: 105-1.37. STRICKLAND, J. D. H. \& PARSON, T. R. 1965. A manual of seawater analysis. Bulletim Fisheries Research Board of Canada. 125: $1-205$

UNESCO. 1973._International oceanographic tables. Wormley. v. 2. VIRNSTEIN, R. W. 1987. Seagrass associated invertebrate communities of Southeastern U. S. A.: A review Symposium on Subtropical-tropical Seagrasses of Southeastern United Stated. Flor: Mar: Res. Pub 42: 89-116. 